\title{
Amoeba-related health risk in drinking water systems: could monitoring of amoebae be a complementary approach to current quality control strategies?
} Francesc Codony*, Leonardo Martín Pérez, Bárbara Adrados, Gemma Agustí,
Mariana Fittipaldi \& Jordi Morató

Laboratori de Microbiologia Sanitaria i Mediambiental (MSM-Lab) \& Aquasost - UNESCO Chair ir

Sustainability, Universitat Politècnica de Catalunya (UPC), Edifici Gaia, Pg. Ernest Lluch/Rambla Sant Nebridi, Terrassa - 08222, Barcelona, Spain

*Author for correspondence: Tel.: +34937398328 = Fax: +34937398328 = codony@oo.upc.edu

Culture-based methods for fecal indicator microorganisms are the standard protocol to assess potential health risk from drinking water systems. However, these traditional fecal indicators are inappropriate surrogates for disinfection-resistant fecal pathogens and the indigenous pathogens that grow in drinking water systems. There is now a range of molecular-based methods, such as quantitative PCR, which allow detection of a variety of pathogens and alternative indicators. Hence, in addition to targeting total Escherichia coli (i.e., dead and alive) for the detection of fecal pollution, various amoebae may be suitable to indicate the potential presence of pathogenic amoeba-resisting microorganisms, such as Legionellae. Therefore, monitoring amoeba levels by quantitative PCR could be a useful tool for directly and indirectly evaluating health risk and could also be a complementary approach to current microbial quality control strategies for drinking water systems.

Nowadays, the traditional strategy for drinking water quality assessment is monitoring fecal pollution levels by the determination and quantification of specific bacterial groups using culture-based methods. Despite the continuous improvements in microbiological analysis methods or in finding more ideal pollution indicators [1], the tendency is to consider only fecal pollution as the main public health concern.

Fecal pollution monitoring, combined with a good water disinfection program, have been key elements in the distribution of safe water for decades. However, it is well known that, in some cases, the most used microbial indicators, such as fecal Escherichia coli and enterococci, may be inadequate for health risk assessment, mainly because many measurable pathogens are more resistant to conventional water treatments and can persist significantly longer in the environment than these fecal indicators. For this reason, as complementary approach can be analyzed Clostridium perfringens spores, which are more resistant than most viruses and parasitic protozoa to conventional water treatments and can persist significantly longer in the environment than fecal pollutants. Nevertheless, although $C$. perfringens is also included in the rules of several countries (e.g., Spain), this microbial indicator is not tested with the same frequency as $E$. coli. The failure of single indicator organism measurements to correlate with pathogens suggests that public health is not adequately protected by simple monitoring schemes based on single indicator detection, particularly at the detection limits routinely employed [2]. As an alternative, the tendency in developed countries is to detect new contaminants or emergent pathogens in drinking water [3]. Public health authorities around the world are working hard in this area, and one of the best examples is the EPA Contaminants Candidate Lists (CCL) [4]. EPA CCL (which is now in the third edition, CCL3) is a list of contaminants that are not currently subjected to any proposed or promulgated national primary drinking water regulations and are known or expected to occur in public water systems, and may require some kind of regulation. Previously to the inclusion of a contaminant into the CCL an extensive process of evaluation is necessary. The evaluation process includes three steps: identification of the candidates, screening and classification (peer review process).

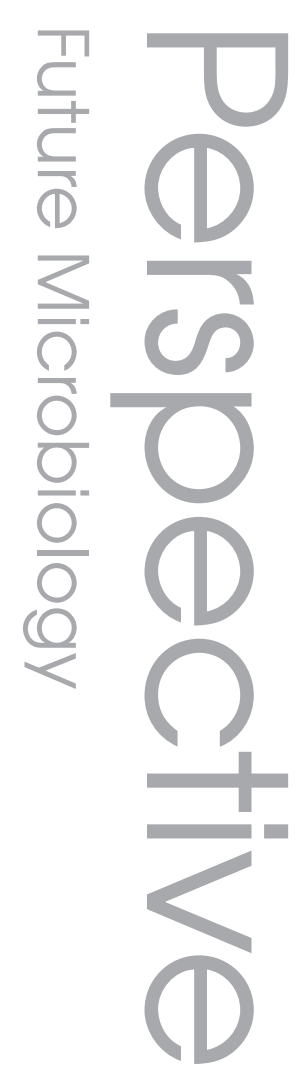

\section{Keywords}

- amoeba $=$ bacterial pathogen = contaminant candidate list $m$ drinking water systems m quantitative PCR

\section{Future Medicine part of}


The development of the CCL is a robust process, and selected agents require evaluation of their potential to cause health impact via drinking water before a determination is made on whether a regulatory control is required or not. The current CCL3 includes four viruses (adenovirus, caliciviruses, enterovirus and Hepatitis A), seven bacteria (Campylobacter jejuni, E. coli O157:H7, Helicobacter pylori, Legionella pneumophila, Mycobacterium avium, Salmonella enterica and Shigella sonnei) and one protozoan (Naegleria fowleri).

The presence of amobae in drinking water systems is not sporadic; they can colonize virtually any kind of water system and support harsh physical and chemical conditions, such as elevated temperature or biocides [5]. It is interesting to note that for all bacteria included in the CCL3, there is published laboratory based evidence of intra-amoebal growth, implying the potential for amoebae to act as environmental vectors and/or transport hosts for the CCL3listed bacterial pathogens. Further evidence is summarized below.

\section{Campylobacterspp.}

Campylobacter spp. are microaerophilic and capnophilic, Gram-negative, curved spiral rodshaped bacteria with a single, unsheathed polar flagellum. Campylobacterspp. are one of the most recognized causative agents of acute gastroenteritis worldwide. C. jejuni is the most frequently isolated species from patients with acute diarrheal disease [6]. They are fecal-borne pathogens and are not particularly resistant to disinfection. For this reason, a conventional indicator such as E. coli is appropriate for evaluating the presence/absence of Campylobacter spp. in drinking water supplies. However, laboratory based studies have demonstrated $C$. jejuni growth at ambient temperatures within amoebae $[7,8]$, opening up the possibility for naturally occurring amoebae to prolong the persistence or even growth of C. jejuni within drinking water systems.

\section{E. coli O157:H7}

E. coli $\mathrm{O} 157: \mathrm{H} 7$ is responsible for a significant proportion of human morbidity and mortality in developed countries [9,10] and is a recurrent source of economic privation for the food industry [11]. E. coli $\mathrm{O} 157: \mathrm{H} 7$ cause diarrhea that ranges from mild and nonbloody to highly bloody, which is indistinguishable from hemorrhagic colitis. Between 2 and 7\% of the cases can further develop into the potentially fatal hemolytic uremic syndrome, characterized by acute renal failure and hemolytic anemia. Children under 5 years of age are at most risk for developing hemolytic uremic syndrome [6] .

A well-publicized waterborne outbreak of illness caused by E. coli O157:H7 (and C. jejuni) occurred in the farming community of Walkerton in Ontario, Canada [6]. At least 16 E. coli $\mathrm{O} 157: \mathrm{H} 7$ outbreaks have been associated with spinach or lettuce contamination since 1995 [101]. One of the most recurrent and crucial questions that emerges from these episodes is how these human pathogens can survive the harsh environmental conditions produced in the field and the sanitation treatments during food processing [12]. The role of protozoa in the survival of E. coli $\mathrm{O} 157: \mathrm{H} 7$ in the natural environment has not been studied; nevertheless, it was demonstrated that E. coli $\mathrm{O} 157: \mathrm{H} 7$ is able to survive and replicate in the frequently encountered environmental protozoan, Acanthamoeba polyphaga [13]. Therefore, protozoa could play a significant role in E. coli O157:H7 survival and transmission [14] and, consequently, may have significant implications for food safety and public health. Survival of E. coli O157:H7 in amoebic cysts may also enhance its distribution, as has been shown for L. pneumophila, Vibrio cholerae and $M$. avium, which may be blown through the air $[15,16]$. Protozoa containing E. coli O157:H7 distributed to grass and silage may subsequently be ingested by grazing cattle and represent a significant vector for the transmission of E. coli O157:H7 [17].

\section{H. pylori}

H. pylori are Gram-negative, microaerobic, curved rod-shaped bacteria responsible for gastric diseases and peptic ulcers, and an important role in gastric cancer and lymphoma. Infection with $H$. pylori is estimated to be $40-70 \%$ worldwide [18]. While $H$. pylori infection sources remain uncertain, recent studies have reported H. pylori DNA detection in water samples [19-22]. This evidence suggests an extensive distribution of $H$. pylori in aquatic environments and may indicate water itself as a potential infection source. However, the survival of Helicobacter and its method of transmission in the natural environment are still unknown.

There is only one published study about the interactions between $H$. pylori and amoebae [23]. In this paper, the authors showed that $H$. pylori was able to survive within trophozoites of Acanthamoeba castellanii under experimental conditions. These authors did not demonstrate the capacity of this bacterium to survive 
in $A$. castellanii cysts. Additional studies are needed to determine this and the role played in vivo by free-living amoebae in the transmission of $H$. pylori, but these findings suggested that amoebae could be a natural reservoir for Helicobacter [24].

\section{M. avium}

$M$. avium are slow-growing, environmental mycobacteria that have been thoroughly studied because they are emerging opportunistic pathogens related to lung diseases and disseminated infections in severely immunocompromised patients $[102,103]$. M. avium have a great capacity to remain in the environment (often found in high numbers in soil and water sources) mainly due to their ability to growth in a wide range of environmental conditions $[102,103]$. In different studies the important role of amoebae in $M$. avium colonization of domestic water supplies has been described [25-27,102,103]. This mycobacterium is capable of survival and growth in Tetrahymena pyriformis, A. polyphaga and $A$. castellanii, implicating these protozoa as possible reservoirs for $M$. avium in the environment $[26,27]$. While L. pneumophila resides within the cysts, $M$. avium was found within the outer walls of the double-walled cysts of $A$. polyphaga. These locations may provide a reservoir for the bacteria when environmental conditions become unfavorable [16]. In addition, the possibility that environmental amoebae could be involved in the mechanisms of pathogenesis of $M$. avium during human infections has been discussed [28].

\section{Salmonella spp.}

Salmonella spp. are motile Gram-negative bacilli belonging to the family Enterobacteriaceae. All enteric pathogens are members of the $S$. enterica species with the exception of $S$. enterica Typhi. Salmonella infections can be divided into four typically clinical manifestations: gastroenteritis, bacteremia or septicemia, typhoid/enteric fever and an asymptomatic carrier state. S. enterica are widely distributed in the environment, but some serovars show host specificity. These pathogens typically get into water systems through fecal contamination from sewage discharges, livestock and wild animal feces. S. enterica are relatively sensitive to disinfection. E. coli is generally a reliable index for $S$. enterica presence in drinking water supplies [6].

Some interesting interaction phenomena between $S$. enterica and different amoeba types have been well demonstrated in several studies $[12,29,30]$. For example, it have been reported a significant bacterial growth inside contractile vacuoles [29], the intracellular replication of $S$. enterica in Acanthamoeba rhysodes [30], and evidence for viable human pathogens multiplication in protozoan vesicles [12]. A recent study has demonstrated that the Salmonella pathogenicity island 2 is highly induced during $S$. enterica (serovar Typhimurium) infection of $A$. polyphaga and is essential for the survival within amoebae [31]. In addition, these authors have suggested that bacterial virulence factors involved in animal and human pathogenesis may have evolved to play other ecological roles. All these works have contributed significantly to the idea that the interaction between bacteria and amoeba plays an important role in bacteria survival in natural environments (even acting as reservoirs).

\section{Legionellae}

Legionellae are intracellular, Gram-negative bacteria ubiquitous in aquatic environments where protozoa are considered to be the natural primary hosts [32]. Legionella can occasionally produce serious infections among humans, including Legionnaires' disease, a respiratory illness associated with inhalation of aerosols [33] generated by man-made water systems [34-36]. Legionella are a food source for protozoa, which abundantly graze on biofilm bacteria [37]. However, Legionella can, under specific conditions, multiply inside the protozoa after phagocytosis. The ability of $L$. pneumophila to survive within the cysts of $A$. polyphaga as a possible mechanism by which the organism evades disinfection and spreads to colonize new environments has been suggested [38]. Fourteen species of amoebae and two species of ciliated protozoa have been identified as potential natural environmental hosts for L. pneumophila [39,40]. Numerous studies have identified amobae as a growth-supporting factor for L. pneumophila [41-44]. However, some authors have questioned these facts and suggested the bacterial consortium within the biofilm as the nutrient supplier to enable extracellular Legionellae growth [45]. Discussion about the necessity of protozoan presence for replication and spread of biofilm-associated L. pneumophila is still ongoing.

\section{Shigella spp.}

Shigella spp. are Gram-negative, nonsporeforming, nonmotile, rod-like members of the bacterial Enterobacteriaceae family. Shigella spp. can cause serious intestinal diseases with a very low dose (10-100 organisms). Globally, over 2 million infections occur each year, resulting 
in approximately 600,000 deaths, predominantly in developing countries. Shigella spp. are relatively sensitive to disinfection. E. coli (or, alternatively, thermotolerant coliforms) is a generally reliable index for the potential presence of Shigella spp. prevalence in drinking water supplies [6]. The relationship between Shigella and amoebae was suggested many years ago [46]. Furthermore, it has been demonstrated that Shigella (and other pathogens) have an increased resistance to chlorine disinfection within protozoa [47]. Recent studies have concluded that amobae could effectively act as an environmental host for Shigella spp., and this relationship could clearly contribute to the transmission and consequent outbreaks associated with this microorganism $[48,49]$.

\section{Discussion}

Although further research regarding bacteriaamoeba interactions is necessary, the current knowledge for all bacteria present in the CCL $[7,13,23,26,30,41,49]$ indicates that amoebae could play a key role in bacterial resistance to disinfection, and survival and spread in drinking water systems. Additionally, bacteria-amoeba interactions suggest that, after a contamination episode, the presence of fecal-related pathogens, such as C. jejuni, E. coli $\mathrm{O} 157: \mathrm{H} 7$, H. pylori, S. enterica and $S$. sonnei, could not be detected using only conventional fecal indicators, as some enteric bacterial pathogens may survive within amoebae and still be present at lower concentrations.

Once again, we face to an old dilemma, the option between detect all health risk-associated indicator microorganisms or find out a new one more appropriate. Furthermore, we have the opinion that public health authorities should rethink health hazard prevention strategies and consider not only microbial pathogens but also include other persistent indicators and potential hosts, such as amoebae.

Most CCL3 pathogens are currently detected by conventional culture-based methods, which require considerable time and expense to detect. Furthermore, in some cases, such as Legionellae or Mycobacterium spp., bacterial culture may require long incubation times, from 7 days up to several weeks.

By contrast, molecular-based methods are available [50,51] and PCR primers and probes can be designed for most microorganisms if not already commercially available. However, despite the rapidity of quantitative PCR (qPCR), it does not generally discriminate between viable and nonviable target microorganisms. In order to overcome this limitation, several studies have recently appeared in which qPCR was used in combination with a sample pretreatment step with propidium monoazide (PMA). PMA is a DNA-intercalating dye with the capacity to only penetrate membrane-compromised cells, blocking the amplification cycle. With this approach, cell viability is based on membrane integrity [52-59].

qPCR combined with PMA could be an adequate method to reduce PCR signals from the DNA of dead cells, allowing the detection and quantification of live pathogens only in environmental samples [52-55]. The use of qPCR in conjunction with PMA has been effectively evaluated in different bacteria, bacterial viruses, bacterial spores and fungi with excellent results [56-58] and is in continuous development. Although viability assessment of human enteric viruses by PCR has not been effectively demonstrated [59], and it is not clear if this technique could be a universal approach for all cases, we bet that this analytical field still has a great potential.

Even if several studies have used qPCR to detect different amoebae species [60-63], the current knowledge suggest that it will be necessary to use different sets of primers (and probes) to detect all the different species. Recently, the use of qPCR and PMA has been positively evaluated by our group in $A$. castellani [64].

As previously noted by other researchers [65], we also believe that more efforts should be directed towards amoebae detection with the aim of use amoeba levels as a complementary health risk indicator. Firstly, because of the previously presented evidence regarding the role of amoebae in bacteria survival; and secondly, because some amobae are also pathogens, such as $N$. fowleri [4] (included in the CCL 3 list) and Acanthamoeba [63]. Additionally, mimivirus, a giant virus that infects amoeba, should also be considered as it has been implicated with various cases of pneumonia [66]. This microorganism also was suggested to be a CCL candidate by the American Society for Microbiology. Regarding the evaluation of amoeba levels, molecular biology will be a useful tool. qPCR could be used for setting amoeba levels and, thus, may help to complement the current quality control strategies directed to minimize the health risk due to pathogen presence, survival or proliferation, throughout drinking water systems.

In our opinion, in the future, microbiological quality control of drinking waters will be based on the combination of conventional microbiology (based in culture) for classical 
indicator detection and molecular biology to target specific pathogens or new indicators. For these reasons, we think that amoebae should be considered as an appropriate candidate for health risk evaluation. Public health authorities should rethink the current prevention strategies for drinking water system assessment and not only consider microbial pathogens, but also include others microorganisms related to pathogen survival.

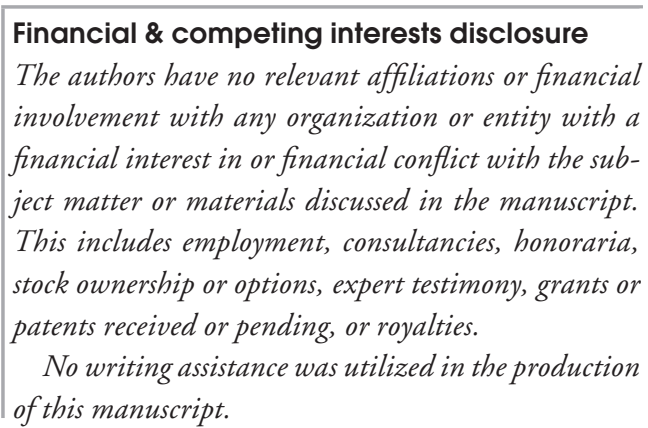

\section{Executive summary}

\section{Amoebae are related to health risk in drinking water systems}

- Amoebae play an active role in survival, spread and/or growth of pathogenic bacteria in drinking water systems.

- Some amobae that are present in drinking water are pathogenic.

Amoebae monitoring could be a complementary approach to current quality control strategies

- Molecular methods allow the detection and quantification of amoeba levels.

- The presence of amoebae indicates possible pathogen survival or regrowth.

\section{Conclusion}

- Health risks associated with fecal pollution may not correlate with health risks related to the presence of amoeba.

- Monitoring amoeba levels may complete the current strategies for drinking water quality surveillance, avoiding the need to analyze the presence of specific microorganisms.

\section{References}

1. Yates MV. Classical indicators in the 21st century-far and beyond the coliform. Water Environ. Res. 79(3), 279-286 (2007).

2. Harwood VJ, Levine AD, Scott TM et al. Validity of the indicator organism paradigm for pathogen reduction in reclaimed water and public health protection. Appl. Environ. Microbiol. 71(6), 3163-3170 (2005).

3. Leclerc H, Scheartzbrod L, Dei-Cas E. Microbial agents associated with waterborne diseases. Crit. Rev. Microbiol. 28(4), 371-409 (2002).

4. US-environmental protection agency. Drinking water contaminant candidate list 3-final. EPA-HQ-OW-2007-1189 FRL-8963-8966. Federal Register 74(194), 51850-51862 (2009).

-5. Thomas V, McDonnell G, Denyer SP, Maillard JY. Free-living amoebae and their intracellular pathogenic microorganisms: risks for water quality. FEMS Microbiol. Rev. 34(3), 231-259 (2009).

6. WHO: guidelines for drinking-water quality: microbial fact sheets (Chapter 11). World Health Organization, Geneva, Switzerland (2008).

7. Axelsson-Olsson D, Waldenström J, Broman T et al. Protozoan Acanthamoeba polyphaga as a potential reservoir for Campylobacter jejuni. Appl. Environ. Microbiol. 71(2), 987-992 (2005).
-8. Snelling WJ, McKenna JP, Lecky DM et al. Survival of Campylobacter jejuni in waterborne protozoa. Appl. Environ. Microbiol. 71(9), 5560-5571 (2005).

9. Besser RE, Griffin PM, Slutsker L. Escherichia coli O157:H7 gastroenteritis and the hemolytic uremic syndrome: an emerging infectious disease. Ann. Rev. Med. 50, 355-367 (1999).

10. Rangel JM, Sparling PH, Crowe C et al. Epidemiology of Escherichia coli O157:H7 outbreaks, United States, 1982-2002. Emerg. Infect. Dis. 11, 603-609 (2005).

11. DeWaal C, Johnson K, Bhuiya F. Outbreak alert! Closing gaps in our federal food safety net. In: Center for Science Public Interest. Center for Science Public Interest, Washington, DC, USA, 2006.

12. Gourabathini P, Brandl MT, Redding KS et al. Interactions between food-borne pathogens and protozoa isolated from lettuce and spinach. Appl. Environ. Microbiol. 74, 2518-2525 (2008).

13. Barker J, Humphrey TJ, Brown MRW. Survival of Escherichia coli O157 in environmental protozoa: implications for disease. FEMS Microbiol. Lett. 173, 291- 295 (1999).

14. Steingberg KM, Levin BR. Grazing protozoa and the evolution of the Escherichia coli O157:H7 Shiga toxin-encoding prophage. Proc. R. Soc. B. 274, 1921-1929 (2007).

15. Brown MRW, Barker J. Unexplored reservoirs of pathogenic bacteria: protozoa and biofilms. Trends Microbiol. 7, 45-50 (1999).
16. Steinert M, Birkness K, White E, Fields B, Quinn F. Mycobacterium avium bacilli grow saprozoically in coculture with Acanthamoeba polyphaga and survive within cyst walls. Appl. Environ. Microbiol. 64, 2256-2261 (1998).

17. Barker J, Humphrey TJ, Brown MWR. Survival of Escherichia coli 0157 in a soil protozoan: implications for disease. FEMS Microbiol. Lett. 173, 291-295 (1999).

18. Brown LM. Helicobacter pylori: epidemiology and routes of transmission. Epidemiol. Rev. 22(2), 283-297 (2000).

19. Kawaguchi K, Matsu J, Osak T, Kamiya S, Yamaguchi H. Prevalence of Helicobacter and Acanthamoeba in natural environment. Lett. Appl. Microbiol. 48, 465-471 (2009).

20. Nayak AK, Rose JB. Detection of Helicobacter pylori in sewage and water using a new quantitative PCR method with SYBR green. J. Appl. Microbiol. 103(5), 1931-1941 (2007).

21. Voytek MA, Ashen JB, Fogarty LR, Kirshtein JD, Landa ER. Detection of Helicobacter pylori and fecal indicator bacteria in five north American rivers. J. Water Health 3, 405-422 (2005).

22. Baker KH, Hegarty JP. Presence of Helicobacter pylori in drinking water is associated with clinical infection. Scand. J. Infect. Dis. 33(10), 744-746 (2001).

23. Winiecka-Krusnell J, Wreiber K, Euler AV, Engstrand L, Linder E. Free-living amoebae promote growth and survival of Helicobacter pylori. Scand. J. Infect. Dis. 34, 253-256 (2002). 
24. Greub G, Raoult D. Microorganisms resistant to free-living amoebae. Clin. Microbiol. Rev. 17(2), 413-433 (2004).

25. Cirillo JD, Falkow S, Tompkins LS, Bermudez LE. Interaction of Mycobacterium avium with environmental amoebae enhances virulence. Infect. Immun. 65, 3759-3767 (1997).

26. Strahl ED, Gillaspy GE, Falkinham 3rd. Fluorescent acid-fast microscopy for measuring phagocytosis of Mycobacterium avium, Mycobacterium intracellular, and Mycobacterium scrofulaceum by Tetrahymena pyriformis and their intracellular growth. Appl. Environ. Microbiol. 67(10), 4432-4439 (2001).

27. Salah IB, Ghigo E, Drancourt M. Free-living amoebae, a training field for macrophage resistance of mycobacteria. Clin. Microbiol. Infect. 15(10), 894-905 (2009).

28. Miltner EC, Bermudez LE. Mycobacterium avium grown in Acanthamoeba castellanii is protected from the effects of antimicrobials. Antimicrob. Agents Chemother. 44(7), 1990-1994 (2000).

29. Gaze WH, Burroughs N, Gallagher MP, Wellington EMH. Interactions between Salmonella typhimurium and Acanthamoeba polyphaga. Observation of a new mode of intracellular growth within contractile vacuoles. Microb. Ecol. 46, 358-369 (2003).

30. Tezcan-Merdol D, Ljungström M, Winiecka-Krusnell J, Linde E, Engstrand L, Rhen M. Uptake and replication of Salmonella enterica in Acanthamoeba rhysodes. Appl. Environ. Microbiol. 70(6), 3706-3714 (2004).

31. Bleasdale B, Lott PJ, Jaganathan A, Stevens MP, Birtles RJ, Paul W. The Salmonella pathogenicity island (SPI)-2-encoded Type 3 secretion system is essential for survival of Salmonella enterica serovar Typhimurium in free-living amoebae. Appl. Environ. Microbiol. 75(6), 1793-1795 (2009).

32. Thomas V, Herrera-Rimann K, Blanc DS et al. Biodiversity of amoebae and amoebaresisting bacteria in a hospital water network. Appl. Environ. Microbiol. 72, 2428-2438 (2006).

33. Fraser DW. Legionellosis. Evidence of airborne transmission. Ann. N. Y. Acad. Sci. 353, 61-66 (1980).

34. Fry AM, Rutman M, AllanT et al. Legionnaires' disease outbreak in an automobile engine manufacturing plant. J. Infect. Dis. 187, 1015-1018 (2003).

35. O'Loughlin RE, Kightlinger L, Werpy MC et al. Restaurant outbreak of Legionnaires' disease associated with a decorative fountain: an environmental and case control study. BMC Infect. Dis. 9, 87-93 (2007).
36. Engelhart S, Pleischla S, Luck C et al. Hospital-acquired legionellosis originating from a cooling tower during a period of thermal inversion. Int. J. Hyg. Environ. Health 211, 235-240 (2008).

37. Huws SA, McRain AJ, Gilbert P. Protozoan grazing and its impact upon population dynamics in biofilm communities. J. Appl. Microbiol. 98, 238-244 (2005).

38. Kilvington S, Price J. Survival of Legionella pneumophila within cysts of Acanthamoeba polyphaga following chlorine exposure. J. Appl. Microbiol. 68, 519-525 (1990).

39. Fields B. The molecular ecology of Legionellae. Trends Microbiol. 4, 286-290 (1996).

40. Fields BS, Benson RF, Besser RE. Legionella and Legionnaires' disease: 25 years of investigation. Clin. Microbiol. Rev. 15, 506-526 (2002).

41. Fields BS, Sanden GN, Barbaree JM et al. Intracellular multiplication of Legionella pneumophila in amoebae isolated from hospital hot water tanks. Curr. Microbiol. 18, 131-137 (1989).

42. Wadowsky RM, Wilson TM, Kapp NJ et al. Multiplication of Legionella spp. in tap water containing Hartmannella veriformis. Appl. Environ. Microbiol. 57, 1950-1955 (1991).

43. Kuchta JM, States SJ, Wadowsky RM et al. Interactions of Legionella pneumophila with Harmanella veriformis including the efficacy of chlorine or cooper and silver ions to disrupt the intra-amoebic multiplication of Legionella pneumophila. Recent Res. Dev. Microbiol. 2, 405-425 (1998).

44. Murga R, Forster S, Brown E et al. Role of biofilms in the survival of Legionella pneumophila in a model potable-water system. Microbiology 147, 3121-3126 (2001).

45. Temmerman R, Boon N, De Bruyne K et al. Persistence and necrotrophic growth of Legionella pneumophila in the absence of protozoa and biofilms. Appl. Environ. Microbiol. 72, 4323-4328 (2006).

-46. Bracha R, Kobiler D, Mirelman D. Attachment and ingestion of bacteria by trophozoites of Entamoeba histolytica. Infect. Immun. 36(1), 396-406 (1982).

47. King CH, Shotts EB, Wooley RE, Porter KG Survival of coliforms and bacterial pathogens within protozoa during chlorination. Appl. Environ. Microbiol. 54(12), 3023-3033 (1988).

48. Jeong HJ, Jang ES, Han BI et al. Acanthamoeba: could it be an environmental host of Shigella? Exp. Parasitol. 115(2), 181-186 (2007).
49. Saeed A, Abd H, Edvinsson B, Sandström G. Acanthamoeba castellanii an environmental host for Shigella dysenteriae and Shigella sonnei. Arch. Microbiol. 191(1), 83-88 (2008).

50. Savichtcheva O, Okabe S. Alternative indicators of fecal pollution. Relations with pathogens and conventional indicators, current methodologies for direct pathogen monitoring and future application perspectives. Water Res. 40(13), 2463-2476 (2006).

-51. Girones R, Ferru MA, Alonso JL et al. Molecular detection of pathogens in water - the pros and cons of molecular techniques. Water Res. 44, 4325-4339 (2010).

-52. Nocker A, Sossa-Fernandez P, Burr MD, Camper AK. Use of propidium monoazide for live/dead distinction in microbial ecology. Appl. Environ. Microbiol. 73(16), 5111-5117 (2007).

53. Nocker A, Camper AK. Novel approaches toward preferential detection of viable cells using nucleic acid amplification techniques. FEMS Microbiol. Lett. 291(2), 137-142 (2009).

54. Luo JF, Lin WT, Guo Y. Method to detect only viable cells in microbial ecology. Appl. Microbiol. Biotechnol. 86(1), 377-384 (2010).

-55. Fittipaldi M, Codony F, Adrados B, Camper AK, Morató J. Viable real-time PCR in environmental samples: can all data be interpreted directly? Microb. Ecol. 61(1), 7-12 (2011).

-56. Vesper S, McKinstry C, Hartmann C, Neace M, Yoder S, Vesper A. Quantifying fungal viability in air and water samples using quantitative PCR after treatment with propidium monoazide (PMA). J. Microbiol. Meth. 72, 180-184 (2008).

57. Rawsthorne H, Dock CN, Jaykus LA. PCR-based method using propidium monoazide to distinguish viable from nonviable Bacillus subtilis spores. Appl. Environ. Microbiol. 75(9), 2936-2939 (2009).

58. Fittipaldi M, Rodriguez NJ, Codony F, Adrados B, Peñuela GA, Morató J. Discrimination of infectious bacteriophage T4 virus by propidium monoazide real-time PCR. J. Virol. Methods 168(1-2), 228-232 (2010).

Parshionikar S, Laseke I, Fout GS. Use of propidium monoazide in reverse transcriptase PCR to distinguish between infectious and noninfectious enteric viruses in water samples. Appl. Environ. Microbiol. 76(13), 4318-4326 (2010).

60. Khan NA. Acanthamoeba: biology and increasing importance in human health. FEMS Microbiol. Rev. 30, 564-595 (2006). 
61. Pélandakis M, Pernin P. Use of multiplex PCR and PCR restriction enzyme analysis for detection and exploration of the variability in the free-living amoeba Naegleria in the environment. Appl. Environ. Microbiol. 68(4), 2061-2065 (2002).

62. Behets J, Declerck P, Delaedt Y, Verelst L, Ollevier F. Quantitative detection and differentiation of free-living amoeba species using SYBR green-based real-time PCR melting curve analysis. Curr. Microbiol. 53(6), 506-509 (2006).

63. Qvarnstrom Y, Visvesvara GS, Sriram R, da Silva AJ. Multiplex real-time PCR assay for simultaneous detection of Acanthamoeba spp., Balamuthia mandrillaris, and Naegleria fowleri. J. Clin. Microbiol. 44(10), 3589-3595 (2006).
64. Fittipaldi M, Pino Rodriguez NJ, Adrados B et al. Discrimination of viable Acanthamoeba castellani trophozoites and cysts by propidium monoazide real-time polymerase chain reaction. J. Eukaryot. Microbiol. 58(4), 359-364 (2011).

65. Thomas JM, Ashbolt NJ. Do free-living amoebae in treated drinking water systems present an emerging health risk? Environ. Sci. Technol. 45(3), 860-869 (2011).

66. La Scola B, Marrie TJ, Auffray JP, Raoult D. Mimivirus in pneumonia patients. Emerg. Infect. Dis. 11(3), 449-452 (2005).

\section{Website}

101. U.S. Department of agriculture/Agricultural research service: USDA funds research on prevention and control of E. coli $\mathrm{O} 157: \mathrm{H} 7$ in fresh produce. US Department of agriculture/Agricultural research service, Washington, DC.

www.ars.usda.gov/news/news.htm? modecode 53-25-21-00

102. Pathogenic mycobacteria in water: A Guide to Public Health Consequences, Monitoring and Management (Chapter 3). World Health Organization, Geneva (2004) www.who.int/water_sanitation_health/ emerging/en/patmycrobact3.pdf

103. Pathogenic mycobacteria in water: A Guide to Public Health Consequences, Monitoring and Management (Chapter 2). World Health Organization, Geneva (2004) www.who.int/water_sanitation_health/ emerging/en/patmycrobact2.pdf 